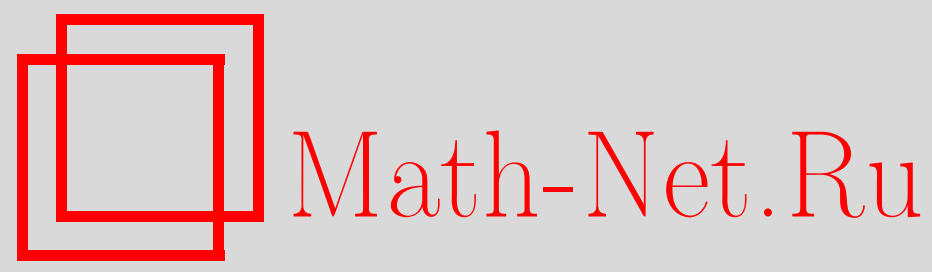

М. И. Толовиков, Распределение некоторых характеристик квазимонотонного отображения, Дискрет. матем., 2010, том 22, выпуск 3, 8-19

DOI: https://doi.org/10.4213/dm1103

Использование Общероссийского математического портала Math-Net.Ru подразумевает, что вы прочитали и согласны с пользовательским соглашением http://www.mathnet.ru/rus/agreement

Параметры загрузки:

IP : 34.229 .108 .108

26 апреля 2023 г., $13: 11: 13$ 
УДК 519.2

\title{
Распределение некоторых характеристик квазимонотонного отображения
}

\author{
() 2010 г. М. И. Толовиков
}

\begin{abstract}
Рассматриваются отображения дискретной окружности в себя, сохраняющие циклический порядок внутри троек точек. Описано строение графа такого отображения, изучено распределение числа и длины его циклов.

Работа выполнена при поддержке Российского фонда фундаментальных исследований, проект 08-01-00078а, и программы Президента Российской Федерации поддержки ведущих научных школ, проект НШ 4129.2006.1.
\end{abstract}

\section{1. Введение}

Будем рассматривать отображения множества $[n]=\{0,1, \ldots, n-1\}$ в себя, которые, следуя В. А. Ватутину и А. М. Зубкову, будем называть квазимонотонными. Формальное определение дадим в терминах графов.

Пусть $C_{n}$ - ориентированный граф с множеством вершин $[n]$. Дуга $(p, q)$ принадлежит орграфу $C_{n}$ тогда и только тогда, когда $q=(p+1) \bmod n$. Простая цепь $[x, y]$ в орграфе $C_{n}$ - это последовательность попарно различных вершин $v_{1}=x, v_{2}, \ldots, v_{k}=y$ такая, что $k<n$ и $\left(v_{i}, v_{i+1}\right)$ есть дуга орграфа $C_{n}$ для всех $i=1,2, \ldots, k-1$. Последовательность, состоящую из одной вершины, и пустую последовательность также будем называть цепями. Удобно считать, что точки $0,1, \ldots, n-1$ расположены на окружности в порядке движения часовой стрелки. Тогда цепь $[x, y]-$ последовательность точек, проходимых при движении от $x$ к $y$ по направлению движения часовой стрелки. Множество вершин цепи $[x, y]$ мы также будем обозначать символом $[x, y]$ и будем называть это множество цепью.

Определение 1. Отображение $f:[n] \rightarrow[n]$ называется квазимонотонным, если для любых $i, j \in[n]$ множество $f^{-1}([i, j])$ является простой цепью в орграфе $C_{n}$.

Пример 1. В табл. 1 представлено квазимонотонное отображение $f:[15] \rightarrow[15]$.

Б. А. Севастьяновым в [1] введено понятие отображения дискретной окружности в себя, сохраняющего ориентацию, близкое к понятию квазимонотонного отображения. Именно, определена функция $R_{0}$, которая каждой упорядоченной тройке точек $(x, y, z)$ на окружности ставит в соответствие элемент множества $\{-1,0,1\}$, причем $R_{0}(x, y, z)=1$, когда точки $x, y, z$ различны и расположены на окружности в порядке движения часовой стрелки, $R_{0}(x, y, z)=-1$, когда точки $x, y, z$ различны и идут на окружности против часовой стрелки, $R_{0}(x, y, z)=0$, когда хотя бы две из точек $x, y, z$ совпадают. Отображение $f$ дискретной окружности в себя называется сохраняющим ориентацию 
Таблица 1. Квазимонотонное отображение $f:[15] \rightarrow[15]$

\begin{tabular}{c|ccccccccccccccc}
$i$ & 0 & 1 & 2 & 3 & 4 & 5 & 6 & 7 & 8 & 9 & 10 & 11 & 12 & 13 & 14 \\
\hline$f(i)$ & 3 & 6 & 7 & 7 & 8 & 8 & 13 & 13 & 13 & 14 & 14 & 0 & 0 & 3 & 3
\end{tabular}

тогда и только тогда, когда для любых трех точек $x, y, z$ из $R_{0}(x, y, z)=1$ следует, что $R_{0}(f(x), f(y), f(z)) \geqslant 0$.

Связь понятий квазимонотонного отображения и отображения, сохраняющего ориентацию, следующая: любое отображение, принимающее не более двух значений, сохраняет ориентацию; если же отображение $f$ принимает более двух различных значений, то $f$ сохраняет ориентацию тогда и только тогда, когда оно квазимонотонно.

Основной известный результат о строении графа квазимонотонного отображения состоит в том, что все циклы такого отображения (и отображения, сохраняющего ориентацию) имеют одну и ту же длину. Б. А. Севастьянов в [1] указывает, что это утверждение было доказано в 1981 г. И. И. Гезенко и в 1984 г. В. А. Ватутиным и А. М. Зубковым, и приводит новое доказательство. Данное явление известно в теории динамических систем для случая непрерывной окружности (см. [2], гл. 11).

Изложим теперь план статьи. В первом разделе описано строение графа квазимонотонного отображения. Построена биекция между множеством таких графов, имеющих заданное строение циклической части, и множеством циклических последовательностей упорядоченных пар плоских корневых деревьев с выделенной вершиной одного из деревьев. В разделе 2 эта биекция использована для подсчета числа квазимонотонных отображений множества $[n]$ в себя, имеющих заданное число циклических точек, заданное число и длину циклов. В разделе 3 описано предельное поведение при $n \rightarrow \infty$ распределения числа циклических точек, числа и длины циклов случайного квазимонотонного отображения из $[n]$ в $[n]$ при равномерном распределении на множестве таких отображений.

Автор благодарит В. А. Ватутина за постановку задачи и внимание к работе.

\section{2. Строение графа квазимонотонного отображения}

Отображение $g:[n] \rightarrow[n]$ называется монотонным, если для любых $i, j \in[n]$ из того, что $i<j$ следует, что $g(i) \leqslant g(j)$. Следующая лемма характеризует квазимонотонные отображения как такие, которые представимы в виде композиции циклического сдвига и монотонного отображения.

Лемма 1. Отображение $f:[n] \rightarrow[n]$ квазимонотонно тогда и только тогда, когда существует такое монотонное отображение $g:[n] \rightarrow[n]$ u такое a $\in[n]$, что $f(i)=g((i+a) \bmod n)$ для всех $i \in[n]$.

Доказательство. Если отображение $f$ вырожденное, то есть его образ состоит из одной точки, то оно представимо в требуемом виде. Предположим, что $f$ - невырожденное отображение. Пусть

$$
j_{0}=\min \left\{j \in[n] \mid f^{-1}(j) \neq \varnothing\right\},
$$

$i_{0}$ - такая вершина орграфа $C_{n}$, что $i_{0} \in f^{-1}\left(j_{0}\right)$ и не существует $i \in f^{-1}\left(j_{0}\right)$ такого, что $\left(i, i_{0}\right)$ - дуга орграфа $C_{n}$. Тогда отображение $g:[n] \rightarrow[n], g(k)=f\left(\left(k+i_{0}\right) \bmod n\right)$, является монотонным. Следовательно, $f=g \circ c_{a}$, где $a=\left(-i_{0}\right) \bmod n, c_{a}$ - циклический 
сдвиг на величину $a: c_{a}(i)=(i+a) \bmod n$. Обратно, любая композиция циклического сдвига и монотонного отображения является квазимонотонным отображением, так как

$$
f^{-1}([i, j])=\left\{(p-a) \bmod n \mid p \in g^{-1}([i, j])\right\}
$$

есть простая цепь в орграфе $C$.

Для отображения $f$ из примера $1 j_{0}=0, i_{0}=11, a=4$, отображение $g$ в точках $0,1, \ldots, 14$ принимает значения $0,0,3,3,3,6,7,7,8,8,13,13,13,14,14$ соответственно.

Теорема 1. Пусть $f:[n] \rightarrow[n]-$ квазимонотонное отображение, $x_{0}<x_{1}<\ldots<x_{m-1}$ - все циклические точки отображения $f$. Тогда справедливы следующие утверждения.

(1) Ограничение отображения $f$ на множество $X=\left\{x_{0}, x_{1}, \ldots, x_{m-1}\right\}$ действует как ичикический сдвиг, то есть существует единственное $c \in[\mathrm{m}]$ такое, что $f\left(x_{i}\right)=x_{(i+c) \bmod m}$ для всех $i=0,1, \ldots, m-1$.

(2) Все иикль отображения $f$ имеют одну и ту же длину.

(3) Число ичиклов $k$ отображения $f$ есть наибольший общий делитель с и $m$, а длина циклов д равна $\mathrm{m} / \mathrm{k}$.

Доказательство. Ограничение $\left.f\right|_{X}$ отображения $f$ на множество его циклических точек является квазимонотонным отображением (точки $\left\{x_{0}, x_{1}, \ldots, x_{m-1}\right\}$ отождествляются с их номерами). Действительно, заменив в графе $C_{n}$ каждую цепь, соединяющую $x_{i}$ с $x_{(i+1) \bmod m}$, одной дугой, получим граф $C_{m}$. Поскольку прообраз при отображении $f$ любой цепи в графе $C_{n}$ есть цепь в графе $C_{n}$, прообраз при отображении $\left.f\right|_{X}$ любой цепи в графе $C_{m}$ есть цепь в графе $C_{m}$. Это и означает, что $\left.f\right|_{X}$ квазимонотонно. Следовательно, в силу леммы 1 , существует такое монотонное отображение $g:[m] \rightarrow[m]$ и такое $c \in[m]$, что $f\left(x_{i}\right)=x_{g}((i+c) \bmod m)$. Так как $\left.f\right|_{X}-$ биекция, $g$ - также биекция, а так как $g$ монотонно, $g$ - тождественное отображение.

Наименьшее значение $q \in[m], q \neq 0$, при котором справедливо равенство $(i+q c) \bmod m=i$, есть $d=m / k$, где $k$ - наибольший общий делитель $c$ и $m$. При этом точки $x_{0}, \ldots, x_{k-1}$ принадлежат различным циклам, следовательно, отображение $f$ имеет ровно $k$ циклов длины $d$.

Величину $c$, введенную в п. 1 теоремы 1 , будем называть числом вращения квазимонотонного отображения $f$.

Для отображения из примера 1 циклическими точками являются 3, 7, 13, число вращения $c=3$.

Определение 2. Плоским корневым деревом называется конечный ориентированный граф, в котором одна вершина не имеет выходящих из нее дуг и называется корнем, а каждая вершина, отличная от корня, имеет ровно одну выходящую из нее дугу. При этом для каждой вершины определен линейный порядок на множестве всех вершин, из которых в нее ведут дуги (ее прообразов).

Два плоских дерева называются изоморфными тогда и только тогда, когда существует взаимно однозначное соответствие между множествами их вершин, которое дуги переводит в дуги и сохраняет линейный порядок на множестве всех прообразов каждой вершины. Говоря о числе плоских деревьев, мы будем понимать под словами плоское дерево класс изоморфизма плоских деревьев. 
Плоское дерево можно представлять нарисованным на евклидовой плоскости так, что дуги, входящие в данную вершину, расположены по часовой стрелке в порядке следования их начальных вершин. Два плоских дерева изоморфны тогда и только тогда, когда существует сохраняющий ориентацию гомеоморфизм плоскости на себя, переводящий одно дерево в другое.

Наряду с квазимонотонными отображениями будем рассматривать комбинаторные конфигурации, которые назовем корневыми квазимонотонными отображениями.

Определение 3. Корневым квазимонотонным отображением с корнем $a$ называется упорядоченная пара $(f, a)$, в которой $f:[n] \rightarrow[n]-$ квазимонотонное отображение, $a \in[n]$ таково, что отображение $g:[n] \rightarrow[n], g(i)=f((i-a) \bmod n)$, монотонно.

Согласно лемме 1 , для любого квазимонотонного отображения $f:[n] \rightarrow[n]$ существует такое $a$, что отображение $g:[n] \rightarrow[n], g(i)=f((i-a) \bmod n)$, монотонно. Если отображение $f$ не является вырожденным, то есть принимает хотя бы два различных значения, то такое $a$ единственно. Поэтому существует взаимно однозначное соответствие $f \mapsto(f, a)$ между множеством невырожденных квазимонотонных отображений $f:[n] \rightarrow[n]$ и множеством корневых квазимонотонных отображений $(f, a)$, в которых $f$ принимает хотя бы два значения. Если же отображение $f:[n] \rightarrow[n]$ принимает лишь одно значение, то при любом $a \in[n]$ отображение $g:[n] \rightarrow[n], g(i)=f((i-a) \bmod n)$, монотонно. Следовательно, корневых квазимонотонных отображений $(f, a)$, в которых отображение $f:[n] \rightarrow[n]$ вырождено, в $n$ раз больше, чем вырожденных квазимонотонных отображений $f:[n] \rightarrow[n]$.

Обозначим $M_{n, m, c}$ множество всех корневых квазимонотонных отображений $(f, a)$ таких, что отображение $f:[n] \rightarrow[n]$ имеет $m$ циклических точек и число вращения $c \in[m]$.

Циклической последовательностью назовем любую последовательность элементов, находящихся во взаимно однозначном соответствии с точками (дискретной) окружности. Две такие последовательности считаем одинаковыми тогда и только тогда, когда они совмещаются поворотом окружности.

Обозначим через $C T_{n, m}$ множество всех циклических последовательностей вида $\left(t_{i}^{1}, t_{i}^{2}\right), i=0,1, \ldots, m-1$, удовлетворяющих условиям:

(1) для каждой пары, входящей в последовательность, ее элементы $t_{i}^{1}, t_{i}^{2}$ есть плоские корневые деревья, имеющие общий корень и не имеющие других общих вершин;

(2) деревья, входящие в различные пары, общих вершин не имеют;

(3) ровно одна вершина из множества вершин всех деревьев $t_{i}^{1}, t_{i}^{2}, i=0,1, \ldots, m-1$, является выделенной.

Удобно считать, что корни деревьев $t_{i}^{1}, t_{i}^{2}$ расположены на дискретной окружности длины $m$ и пары $\left(t_{i}^{1}, t_{i}^{2}\right)$ находятся во взаимно однозначном соответствии с корнями.

Последовательности из $C T_{n, m}$ мы также рассматриваем с точностью до изоморфизма в следующем смысле: две такие последовательности считаем изоморфными, если существует биекция множества вершин всех деревьев одной последовательности на аналогичное множество вершин второй последовательности, осуществляющая одновременно поворот окружности, на которой расположены корни деревьев, изоморфизм всех соответствующих плоских деревьев, и переводящая выделенную вершину в выделенную.

Теорема 2. При любых фиксированных $n \in \mathbf{N}, 1 \leqslant m \leqslant n-1, c \in[m]$ существует взаимно однозначное соответствие между элементами множеств $M_{n, m, c}$ и $C T_{n, m}$. 
Доказательство. Пусть $(f, a) \in M_{n, m, c}-$ корневое квазимонотонное отображение, в котором $f:[n] \rightarrow[n]-$ квазимонотонное отображение с заданным числом вращения $c$, и пусть $x_{0}, x_{1}, \ldots, x_{m-1}$ - все циклические точки отображения $f$. В силу определения 1 , при любом $i \in[m]$ множество $f^{-1}\left(x_{i}\right)=f^{-1}\left(\left[x_{i}, x_{i}\right]\right)$ есть простая цепь $\left[y_{i}, z_{i}\right]$ в орграфе $C_{n}$. Это множество содержит ровно одну циклическую точку $r_{i}=\left(\left.f\right|_{X}\right)^{-1}\left(x_{i}\right)$ отображения $f$. Если отображение $f$ невырождено, то вершина $r_{i}$ делит цепь $\left[y_{i}, z_{i}\right]$ на два подмножества: $\left[y_{i}, r_{i}\right)=\left[y_{i}, r_{i}\right] \backslash\left\{r_{i}\right\}$ и $\left(r_{i}, z_{i}\right]=\left[r_{i}, z_{i}\right] \backslash\left\{r_{i}\right\}$. Если же отображение $f$ вырождено, то $f^{-1}\left(x_{i}\right)=f^{-1}\left(x_{0}\right)=[n]$. В этом случае положим $\left[y_{i}, r_{i}\right)=\left[a, x_{0}\right),\left(r_{i}, z_{i}\right]=\left(x_{0}, a\right)$, где $a$ - корень отображения $(f, a)$. Поставим в соответствие вершине $x_{i}$ упорядоченную пару плоских деревьев $\left(t_{i}^{1}, t_{i}^{2}\right)$ с корнем $x_{i}$. Множество вершин дерева $t_{i}^{1}$ есть $\left\{x_{i}\right\} \cup\left(\bigcup_{k \geqslant 0}\left(f^{k}\right)^{-1}\left(\left[y_{i}, r_{i}\right)\right)\right)$, где $f^{k}$ обозначает $k$-ю итерацию отображения $f, f^{0}-$ тождественное отображение. Множество вершин дерева $t_{i}^{2}$ есть $\left\{x_{i}\right\} \cup\left(\bigcup_{k \geqslant 0}\left(f^{k}\right)^{-1}\left(\left(r_{i}, z_{i}\right]\right)\right)$. Дуга из вершины $p$, отличной от корня, в вершину $q$ существует тогда и только тогда, когда $f(p)=q$. Прообразы вершины $p$ упорядочены в соответствии с линейным порядком вершин цепи $f^{-1}(p)$. Наконец, объявим выделенной вершину 0. Таким образом, каждому корневому отображению $(f, a) \in M_{n, m, c}$ поставлена в соответствие циклическая последовательность $\left(t_{i}^{1}, t_{i}^{2}\right), i=0,1, \ldots, m-1$, из множества $C T_{n, m}$.

Обратно, пусть задана циклическая последовательность $\left(t_{i}^{1}, t_{i}^{2}\right), i=0,1, \ldots, m-1$, из множества $C T_{n, m}$. Построим корневое квазимонотонное отображение $(f, a)$ с заданным числом вращения $c \in[m]$, которому соответствует последовательность пар деревьев $\left(t_{i}^{1}, t_{i}^{2}\right), i=0,1, \ldots, m-1$, в смысле соответствия, описанного в первой части доказательства. Отметим на окружности $m$ точек $x_{0}, x_{1}, \ldots, x_{m-1}$, следующих друг за другом в порядке движения часовой стрелки (пока не определено, каким элементам множества $[n]$ соответствуют эти точки). Объявим точку $x_{i}$ корнем деревьев $t_{i}^{1}, t_{i}^{2}$ и положим $f\left(x_{i}\right)=x_{(i+c) \bmod m}, i=0,1, \ldots, m-1$. Назовем высотой вершины дерева число дуг ориентированной цепи, ведущей из этой вершины в корень. Обозначим через $t_{i}^{j}(k)$ множество вершин высоты $k$ в дереве $t_{i}^{j}$. На множестве $t_{i}^{j}(k)$ определим линейный порядок. При $k=0$ это множество состоит из одного корня. Если линейный порядок на множестве $t_{i}^{j}(k)$ уже определен, то упорядочим вершины множества $t_{i}^{j}(k+1)$ так: прообразы одной и той же вершины множества $t_{i}^{j}(k)$ упорядочиваются как в рассматриваемом плоском дереве; если же из вершин $u$ и $v$ ведут дуги в различные вершины $u^{\prime}$ и $v^{\prime}$ множества $t_{i}^{j}(k)$, то $u$ предшествует $v$ тогда и только тогда, когда $u^{\prime}$ предшествует $v^{\prime}$. Расположим теперь на окружности все некорневые вершины деревьев $t_{i}^{1}, t_{i}^{2}, i=0,1, \ldots, m-1$. На дуге $\left[x_{i}, x_{i+1}\right]$ (если $m=1$, то $\left.\left[x_{i}, x_{i+1}\right]=[n]\right)$ вслед за точкой $x_{i}$ в порядке движения часовой стрелки последовательно отметим вершины множеств $t_{(i+q c) \bmod m}^{2}(q), q=1,2, \ldots, n-1$, и $t_{(i+1+p c) \bmod m}^{1}(p), p=n-1, \ldots, 1,2$, соблюдая порядок вершин внутри каждого из этих множеств (если множество пусто, то ничего не отмечаем). Дуги деревьев при расположении их вершин на окружности сохраним. Пронумеруем отмеченные на окружности точки элементами множества $[n]$ в порядке движения часовой стрелки так, чтобы выделенная в одном из деревьев вершина получила номер 0. Положим $f(r)=s$, если из точки с номером $r$ ведет дуга в точку с номером $s$. Теперь отображение $f$ определено полностью. Если $f$ невырождено, то $a$ и соответствующее корневое отображение $(f, a)$ определяются по $f$ однозначно. Если же $f$ вырождено, то положим $a$ равным метке первой в порядке расположения на окружности вершины дерева $t_{0}^{1}$.

Теорема доказана.

На рис. 1 представлен граф квазимонотонного отображения $f$, рассмотренного выше, и показаны деревья $t_{i}^{1}, t_{i}^{2}, i=0,1, \ldots, 2$. Лучи, исходящие из циклических вершин, 


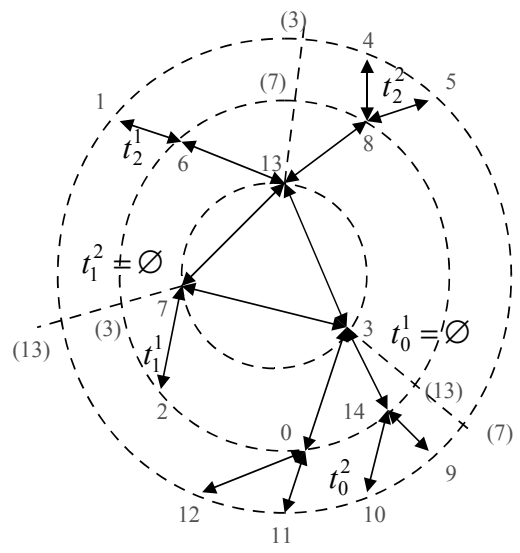

Рис. 1.

изображают траектории этих точек при итерациях отображения $\left(\left.f\right|_{X}\right)^{-1}$. На каждой концентрической окружности лежат вершины деревьев $t_{i}^{1}, t_{i}^{2}$, имеющие одну и ту же высоту над корнем.

\section{3. Число квазимонотонных отображений}

Введем следующие обозначения. Пусть $Q(n)$ - число квазимонотонных отображений $f:[n] \rightarrow[n], Q(n, m), Q(n, k, d)$ - число таких отображений, имеющих соответственно $m$ циклических точек и $k$ циклов, длина каждого из которых равна $d$. Символ $R Q$ используем для обозначения числа корневых квазимонотонных отображений с соответствующими ограничениями.

Теорема 3. Справедливы равенства

$$
\begin{array}{r}
R Q(n)=\frac{n}{2}\left(\begin{array}{c}
2 n \\
n
\end{array}\right) ; \quad R Q(n, m)=m\left(\begin{array}{c}
2 n \\
n+m
\end{array}\right) ; \quad R Q(n, k, d)=\varphi(d)\left(\begin{array}{c}
2 n \\
n+k d
\end{array}\right) ; \\
Q(n)=\frac{n}{2}\left(\begin{array}{c}
2 n \\
n
\end{array}\right)-n(n-1) ; \quad Q(n, 1)=n ; \quad Q(n, m)=m\left(\begin{array}{c}
2 n \\
n+m
\end{array}\right), \quad m>1 ; \\
Q(n, 1,1)=1 ; \quad Q(n, k, d)=\varphi(d)\left(\begin{array}{c}
2 n \\
n+k d
\end{array}\right), \quad k d>1 .
\end{array}
$$

Здесь $\varphi-$ функиия Эйлера, $\varphi(d)$ есть количество натуральных чисел, не превосходящих $d$ и взаимно простых с $d$.

Доказательство. Пусть $C_{n}$ обозначает число плоских корневых деревьев, имеющих ровно $n$ вершин, отличных от корня, и

$$
C(x)=\sum_{n=0}^{\infty} C_{n} x^{n}
$$


- производящая функция чисел $C_{n}$. Числа $C_{n}$ называются числами Каталана. Как известHO,

$$
C_{n}=\frac{1}{n+1}\left(\begin{array}{c}
2 n \\
n
\end{array}\right), \quad C(x)=\frac{1-\sqrt{1-4 x}}{2 x} .
$$

Обозначим через $D_{n}$ число упорядоченных пар плоских корневых деревьев, имеющих в сумме $n-1$ некорневых вершин. Тогда производящая функция

$$
D(x)=\sum_{n=1}^{\infty} D_{n} x^{n}
$$

чисел $D_{n}$ равна

$$
D(x)=x C^{2}(x)=C(x)-1 .
$$

Пусть $\left[x^{n}\right] g(x)$ обозначает коэффициент при $x^{n}$ в степенном ряде $g(x)$. Тогда число циклических последовательностей из $m$ пар плоских корневых деревьев, в которых общее число некорневых вершин равно $n-m$, есть $(1 / m)\left[x^{n}\right] D^{m}(x)$. Выделенную вершину одного из этих деревьев можно выбрать $n$ способами, а кручение $c \in[m]-m$ способами, поэтому, согласно теореме 2,

$$
R Q(n, m)=n\left[x^{n}\right] D^{m}(x)
$$

Функция $D(x)$ удовлетворяет уравнению

$$
D(x)=x(D(x)+1)^{2} .
$$

Согласно теореме Лагранжа ([3], с. 26), если функция $w(x)$ является решением уравнения

$$
w(x)=x \psi(w(x))
$$

где $\psi(\lambda)$ - степенной ряд с ненулевым свободным членом, и $f(\lambda)-$ произвольный степенной ряд по неотрицательным степеням $\lambda$, то

$$
\left[x^{n}\right] f(w(x))=\frac{1}{n}\left[\lambda^{n-1}\right]\left(\psi^{n}(\lambda) \frac{d}{d \lambda} f(\lambda)\right) .
$$

Следовательно,

$$
\begin{aligned}
{\left[x^{n}\right](D(x))^{m} } & =\frac{1}{n}\left[\lambda^{n-1}\right]\left((\lambda+1)^{2 n} \frac{d}{d \lambda} \lambda^{m}\right)=\frac{m}{n}\left(\begin{array}{c}
2 n \\
n-m
\end{array}\right)=\frac{m}{n}\left(\begin{array}{c}
2 n \\
n+m
\end{array}\right), \\
R Q(n, m) & =m\left(\begin{array}{c}
2 n \\
n+m
\end{array}\right) .
\end{aligned}
$$

Из третьего утверждения теоремы 1 вытекает, что

$$
R Q(n, k, d)=\frac{\varphi(d)}{m} R Q(n, m)=\varphi(d)\left(\begin{array}{c}
2 n \\
n+m
\end{array}\right)=\varphi(d)\left(\begin{array}{c}
2 n \\
n+k d
\end{array}\right)
$$


Далее,

$$
\begin{aligned}
R Q(n) & =\left[x^{n-1}\right] \sum_{m=1}^{\infty} \frac{d}{d x} D^{m}(x) \\
& =\left[x^{n-1}\right] \frac{d}{d x} \frac{D(x)}{1-D(x)}=\left[x^{n-1}\right] \frac{d}{d x} \frac{1-\sqrt{1-4 x}}{4 x}=\frac{n}{2}\left(\begin{array}{c}
2 n \\
n
\end{array}\right) .
\end{aligned}
$$

На этом доказательство первого ряда равенств в теореме завершено. Для доказательства остальных равенств заметим, что квазимонотонных отображений, принимающих более одного значения, столько же, сколько соответствующих корневых квазимонотонных отображений, а квазимонотонных отображений $f:[n] \rightarrow[n]$, принимающих только одно значение, всего $n$, в то время как соответствующих корневых квазимонотонных отображений $-n^{2}$.

\section{4. Распределение числа циклических точек, числа и длины циклов случайного квазимонотонного отображения}

Пусть на множестве всех квазимонотонных отображений $f:[n] \rightarrow[n]$ задано равномерное распределение вероятностей, и пусть $X$ - число циклов, $Y$ - длина каждого из циклов и $Z=X Y-$ число циклических точек случайного квазимонотонного отображения $f$. Тогда

$$
\begin{aligned}
& \mathbf{P}(Z=m)=\frac{Q(n, m)}{Q(m)}, \\
& \mathbf{P}(X=k, Y=d)=\frac{Q(n, k, d)}{Q(m)}, \\
& \mathbf{P}(X=k)=\sum_{d=1}^{n} \frac{Q(n, k, d)}{Q(m)}, \\
& \mathbf{P}(Y=d)=\sum_{k=1}^{n} \frac{Q(n, k, d)}{Q(m)} .
\end{aligned}
$$

Таким образом, точные формулы для распределений случайных величин $X, Y, Z$ дает теорема 3.

Обратимся к изучению асимптотики распределений случайных величин $X, Y, Z$. Можно определить аналогичные случайные величины для корневых квазимонотонных отображений. Предельные распределения для этих случайных величин совпадают с предельными распределениями для $X, Y, Z$. Поэтому далее мы будем рассматривать корневые квазимонотонные отображения и понимать под $X, Y, Z$ соответствующие характеристики таких отображений.

Теорема 4. При $n \rightarrow \infty$

(1) $\mathbf{P}(X=k, Y \leqslant x \sqrt{n}) \rightarrow 6\left(1-e^{-k^{2} x^{2}}\right) /\left(\pi^{2} k^{2}\right)$ при любых $k \in \mathbf{N}, x>0$;

(2) $n \mathbf{P}(Z=m)-m e^{-m^{2} / n} \rightarrow 0$ равномерно по $m \in 1,2, \ldots, n$;

$$
\begin{aligned}
& \mathbf{P}(Z \leqslant x \sqrt{n}) \rightarrow 1-e^{-x^{2}}, \quad x>0 ; \\
& \mathbf{E} Z=\frac{\sqrt{\pi n}}{2}(1+o(1)), \\
& \mathbf{D} Z=(1-\pi / 4) n(1+o(1)) .
\end{aligned}
$$


(3) $\mathbf{P}(X=k) \rightarrow 6 /\left(\pi^{2} k^{2}\right)$ при любом $k \in \mathbf{N}$;

(4) при любом $x>0$

$$
\mathbf{P}(Y \leqslant x \sqrt{n}) \rightarrow \frac{6}{\pi^{2}} \sum_{k=1}^{\infty} \frac{1-e^{-k^{2} x^{2}}}{k^{2}} .
$$

Доказательство. Докажем утверждение 1. Согласно теореме 3,

$$
\mathbf{P}(X=k, Y \leqslant x \sqrt{n})=\left(\sum_{1 \leqslant d \leqslant x \sqrt{n}} \varphi(d)\left(\begin{array}{c}
2 n \\
n+k d
\end{array}\right)\right) /\left(\frac{n}{2}\left(\begin{array}{c}
2 n \\
n
\end{array}\right)\right)
$$

Из формулы Стирлинга следует, что

$$
\frac{n}{2}\left(\begin{array}{c}
2 n \\
n
\end{array}\right) \sim \frac{2^{2 n} \sqrt{n}}{2 \sqrt{\pi}}
$$

Воспользовавшись нормальным приближением для биномиального распределения, получаем, что при $n \rightarrow \infty$

$$
\sqrt{\frac{n}{2}}\left(\begin{array}{c}
2 n \\
n+k d
\end{array}\right) 2^{-2 n}-\frac{1}{\sqrt{2 \pi}} e^{-(k d)^{2} / n} \rightarrow 0
$$

равномерно относительно целых значений $k d \in\{1,2, \ldots, n\}$. Следовательно,

$$
\mathbf{P}(X=k, Y \leqslant x \sqrt{n})=\frac{2}{n} \sum_{1 \leqslant d \leqslant x \sqrt{n}} \varphi(d) e^{-(k d)^{2} / n}+\frac{2}{n} \sum_{1 \leqslant d \leqslant x \sqrt{n}} \varphi(d) o(1) .
$$

Для второй суммы известна оценка, полученная Дирихле (см. [4], с. 40): при $n \rightarrow \infty$

$$
\sum_{1 \leqslant d \leqslant x \sqrt{n}} \varphi(d)=\frac{3 x^{2}}{\pi^{2}} n+O(x \sqrt{n} \ln (x \sqrt{n})),
$$

откуда

$$
\left.\mathbf{P}(X=k, Y \leqslant x \sqrt{n})=\frac{2}{n} \sum_{1 \leqslant d \leqslant x \sqrt{n}} \varphi(d) e^{-(k d)^{2} / n}\right)+o(1) .
$$

Чтобы оценить первую сумму, используем способ рассуждений Дирихле.

Справедливо равенство (см. [3], с. 30)

$$
\varphi(d)=d \sum_{q \mid d} \frac{\mu(q)}{q},
$$

где $\mu$ - теоретико-числовая функция Мебиуса (она принимает лишь значения $0,1,-1$ ).

Следовательно,

$$
\begin{aligned}
\frac{2}{n} \sum_{1 \leqslant d \leqslant x \sqrt{n}} \varphi(d) e^{-(k d)^{2} / n} & =\frac{2}{n} \sum_{1 \leqslant d \leqslant x \sqrt{n}} d \sum_{q \mid d} \frac{\mu(q)}{q} e^{-(k d)^{2} / n} \\
& =\frac{2}{n} \sum_{1 \leqslant q \leqslant x \sqrt{n}} \mu(q) \sum_{1 \leqslant r \leqslant x \sqrt{n} / q} r e^{-(k q r)^{2} / n} .
\end{aligned}
$$


Сравнивая сумму с интегралом $\int_{0}^{x / q} t e^{-(k q t)^{2}} d t$, получаем, что

$$
\sum_{1 / \sqrt{n} \leqslant r / \sqrt{n} \leqslant x / q} \frac{r}{\sqrt{n}} e^{-(k q)^{2}(r / \sqrt{n})^{2}} \frac{1}{\sqrt{n}}=\frac{1-e^{-(k x)^{2}}}{2(k q)^{2}}+O\left(\frac{1}{k q \sqrt{n}}\right) .
$$

Следовательно,

$$
\begin{aligned}
\frac{2}{n} \sum_{1 \leqslant d \leqslant x \sqrt{n}} \varphi(d) e^{-(k d)^{2} / n} & =\frac{1-e^{-(k x)^{2}}}{k^{2}} \sum_{1 \leqslant q \leqslant x \sqrt{n}} \frac{\mu(q)}{q^{2}}+O\left(\frac{1}{k \sqrt{n}} \sum_{1 \leqslant q \leqslant x \sqrt{n}} \frac{|\mu(q)|}{q}\right) \\
& =\frac{1-e^{-(k x)^{2}}}{k^{2}} \sum_{q=1}^{\infty} \frac{\mu(q)}{q^{2}}+O\left(\frac{\ln (x \sqrt{n})}{k \sqrt{n}}\right)+O\left(\frac{1-e^{-(k x)^{2}}}{k^{2} x \sqrt{n}}\right) \\
& =\frac{6}{\pi^{2}} \frac{1-e^{-(k x)^{2}}}{k^{2}}+O\left(\frac{\ln (x \sqrt{n})}{k \sqrt{n}}\right),
\end{aligned}
$$

где в последнем равенстве мы воспользовались соотношением

$$
\sum_{q=1}^{\infty} \frac{\mu(q)}{q^{2}}=\frac{6}{\pi^{2}}
$$

(см. [4], с. 132). Таким образом, для любого $x>0$ и любого целого $k>0$ при $n \rightarrow \infty$

$$
\mathbf{P}(X=k, Y \leqslant x \sqrt{n})=\frac{6}{\pi^{2}} \frac{1-e^{-(k x)^{2}}}{k^{2}}+o(1) .
$$

Утверждение 1 теоремы доказано.

Докажем утверждение 2. Первое соотношение этого утверждение следует из нормального приближения для биномиального распределения, второе получается из первого. Вычислим первые моменты случайной величины $Z$ :

$$
\begin{aligned}
\mathbf{E} Z & =\left(\frac{n}{2}\left(\begin{array}{c}
2 n \\
n
\end{array}\right)\right)^{-1} \sum_{m=1}^{n} m^{2}\left(\begin{array}{c}
2 n \\
n+m
\end{array}\right) \\
& =\left.\left(\frac{n}{2}\left(\begin{array}{c}
2 n \\
n
\end{array}\right)\right)^{-1} \frac{1}{2}\left(n^{2}(x+y)^{2 n}-x y \frac{d}{d x} \frac{d}{d y}(x+y)^{2 n}\right)\right|_{x=1, y=1} \\
& =\left(\begin{array}{c}
2 n \\
n
\end{array}\right)^{-1} 2^{2 n-1}=\frac{\sqrt{\pi n}}{2}(1+o(1)) .
\end{aligned}
$$

Дисперсия $Z$ получается аналогично. Вычисления дают

$$
\begin{aligned}
& \mathbf{E} Z^{2}=n \\
& \mathbf{D} Z=n-\left(\begin{array}{c}
2 n \\
n
\end{array}\right)^{-2} 2^{4 n-2}=\left(1-\frac{\pi}{4}\right) n(1+o(1)) .
\end{aligned}
$$

Докажем утверждение 3 теоремы. Пусть $k$ фиксировано. Так как предельная функция в утверждении 1 теоремы непрерывна, сходимость равномерна относительно $x$. Следовательно, переходя к пределу при $x \rightarrow \infty$, получим утверждение 3 теоремы. 
Докажем утверждение 4 теоремы. Оценим вероятность $\mathbf{P}(X=k)$. Справедливы неравенства

$$
\begin{aligned}
\frac{2}{n\left(\begin{array}{c}
2 n \\
n
\end{array}\right)} \sum_{d=1}^{n} d\left(\begin{array}{c}
2 n \\
n+d
\end{array}\right) & =\frac{2}{n\left(\begin{array}{c}
2 n \\
n
\end{array}\right)} \sum_{q=0}^{[n / k]} \sum_{r=1}^{k}(k q+r)\left(\begin{array}{c}
2 n \\
n+k q+r
\end{array}\right) \\
& \geqslant \frac{2}{n\left(\begin{array}{c}
2 n \\
n
\end{array}\right)} \sum_{q=0}^{[n / k]} \sum_{r=1}^{k}(k q+r)\left(\begin{array}{c}
2 n \\
n+k q+k
\end{array}\right) \\
& =\frac{2}{n\left(\begin{array}{c}
2 n \\
n
\end{array}\right)} \sum_{q=0}^{[n / k]} \frac{\left((2 q+1) k^{2}+k\right)}{2}\left(\begin{array}{c}
2 n \\
n+k q+k
\end{array}\right) \\
& \geqslant \frac{k^{2}}{n\left(\begin{array}{c}
2 n \\
n
\end{array}\right)} \sum_{q=1}^{[n / k]} q\left(\begin{array}{c}
2 n \\
n+q k
\end{array}\right)=\frac{k^{2}}{2} \mathbf{P}(X=k) .
\end{aligned}
$$

С другой стороны, в силу соотношения

$$
2^{2 n} /\left(\begin{array}{c}
2 n \\
n
\end{array}\right) \sim \sqrt{\pi n}
$$

при всех достаточно больших $n$ выполнены неравенства

$$
\begin{aligned}
\frac{2}{n\left(\begin{array}{c}
2 n \\
n
\end{array}\right)} \sum_{d=1}^{n} d\left(\begin{array}{c}
2 n \\
n+d
\end{array}\right) & =\frac{2^{2 n+1}}{n\left(\begin{array}{c}
2 n \\
n
\end{array}\right)} \sum_{d=1}^{n} d\left(\begin{array}{c}
2 n \\
n+d
\end{array}\right) 2^{-2 n} \\
& =\frac{2^{2 n}}{n\left(\begin{array}{c}
2 n \\
n
\end{array}\right)} \sum_{d=0}^{2 n}|d-n|\left(\begin{array}{c}
2 n \\
d
\end{array}\right) 2^{-2 n} \\
& =\frac{2^{2 n}}{n\left(\begin{array}{c}
2 n \\
n
\end{array}\right)} \mathbf{E}|\mu-n| \leqslant \frac{2}{\sqrt{n}} \mathbf{E}|\mu-n| \\
& \leqslant \frac{2}{\sqrt{n}} \sqrt{\mathbf{E}(\mu-n)^{2}}<2,
\end{aligned}
$$

где случайная величина $\mu$ распределена по биномиальному закону с параметрами $2 n, 1 / 2$. Таким образом, при всех достаточно больших значениях $n$ для всех $k \in \mathbf{N}$ выполнены неравенства

$$
\mathbf{P}(X=k)<\frac{4}{k^{2}} .
$$

Из полученной оценки следует неравенство

$$
\mathbf{P}(X=k, Y \leqslant x \sqrt{n}) \leqslant \mathbf{P}(X=k) \leqslant \sum_{k=k_{0}+1}^{\infty} \frac{4}{k^{2}}<\frac{4}{k_{0}},
$$

справедливое при всех достаточно больших $n$ и любом $k_{0} \in \mathbf{N}$. При этом

$$
\frac{6}{\pi^{2}} \sum_{k=k_{0}+1}^{\infty} \frac{1-e^{-(k x)^{2}}}{k^{2}}<\frac{1}{k_{0}}
$$


поэтому

$$
\begin{aligned}
\left|\mathbf{P}(Y \leqslant x \sqrt{n})-\frac{6}{\pi^{2}} \sum_{k=1}^{\infty} \frac{1-e^{-k^{2} x^{2}}}{k^{2}}\right| & =\left|\sum_{k=1}^{\infty} \mathbf{P}(X=k, Y \leqslant x \sqrt{n})-\frac{6}{\pi^{2}} \sum_{k=1}^{\infty} \frac{1-e^{-k^{2} x^{2}}}{k^{2}}\right| \\
& <\sum_{k=1}^{k_{0}}\left|\mathbf{P}(X=k, Y \leqslant x \sqrt{n})-\frac{6}{\pi^{2}} \frac{1-e^{-k^{2} x^{2}}}{k^{2}}\right|+\frac{5}{k_{0}} .
\end{aligned}
$$

В силу утверждения 1 теоремы, при любом фиксированном $k_{0}$ значение $n$ можно выбрать настолько большим, что неравенство

$$
\left|\mathbf{P}(X=k, Y \leqslant x \sqrt{n})-\frac{6}{\pi^{2}} \frac{1-e^{-k^{2} x^{2}}}{k^{2}}\right|<\frac{1}{k_{0}^{2}}
$$

выполняется при всех $1 \leqslant k \leqslant k_{0}$. Тогда

$$
\left|\mathbf{P}(Y \leqslant x \sqrt{n})-\frac{6}{\pi^{2}} \sum_{k=1}^{\infty} \frac{1-e^{-k^{2} x^{2}}}{k^{2}}\right|<\frac{6}{k_{0}}
$$

при всех достаточно больших $n$. Поскольку $k_{0}$ произвольно, отсюда следует утверждение 4 теоремы.

\section{Список литературы}

1. Севастьянов Б. А., О связи некоторых структурных характеристик прообразов и образов конечных множеств при некоторых классах преобразований. Труды по дискретной математике (1998) 2, 296-304.

2. Каток А. Б., Хасселблат Б., Введение в современную теорию динамических систем. Факториал, Москва, 1999.

3. Гульден Я., Джексон Д., Перечислительная комбинаторика. Наука, Москва, 1990.

4. Виноградов И. М., Основы теории чисел. ГИТТЛ, Москва-Ленинград, 1952.

Статья поступила 15.10.2008. 